\title{
Identifying Tags Describing Image Contents
}

\author{
Taka Maenishi \\ Kyoto University \\ maenishi@dl.soc.i.kyoto-u.ac.jp
}

\begin{abstract}
On many photo-sharing social media, e.g., Instagram, a user posting a photo can add tags, i.e., words describing it. Tags are used for keyword-based image search. Some tags, however, describe not image contents but some metadata, e.g., camera names. We propose a method of determining which tag is more likely to describe the associated image contents. We determine it based on pairwise comparisons of tags. Given a pair of tags A and B, we compare three sets of images: (1) those associated with A, (2) those associated with $\mathrm{B}$, and (3) those associated with both A and B. If (3) is more similar to (1) than to (2), A is more likely to describe image contents. To compute similarity between two image sets, we use the similarity of their associated tags, instead of their visual similarity. Our experiment with Instagram data shows that our method is effective.
\end{abstract}

\section{KEYWORDS}

tag ranking; image tags; social tagging; image captioning

\section{ACM Reference Format:}

Taka Maenishi and Keishi Tajima. 2019. Identifying Tags Describing Image Contents. In 30th ACM Conference on Hypertext and Social Media (HT '19), September 17-20, 2019, Hof, Germany. ACM, New York, NY, USA, 2 pages. https://doi.org/10.1145/3342220.3344936

\section{INTRODUCTION}

On many photo-sharing social media, such as Instagram, a user posting a photo can add tags, i.e., words describing the photo. Tags are used for keyword-based image search. Some tags, however, describe not the image contents but some metadata, such as camera names. In order to improve the quality of keyword-based search, we need a method of distinguishing these two types of tags.

To distinguish them, we should consider whether the given image has any other tags that are more likely to describe the image contents. For example, an image with tags "flower iPhone" is probably a photo of flowers taken by an iPhone, but an image with tags "iPhone new" is probably a photo of an iPhone.

In this paper, we propose a method of determining which of a given pair of tags is more likely to describe the contents of the associated images. It produces a partial ordering (including equivalence classes) among a set of tags associated with a image. We have also developed a method of producing a total ordering based on how many other tags each tag win against in the pairwise comparison, but we leave it out of the scope of this paper for the space limitation.

Permission to make digital or hard copies of part or all of this work for personal or classroom use is granted without fee provided that copies are not made or distributed for profit or commercial advantage and that copies bear this notice and the full citation on the first page. Copyrights for third-party components of this work must be honored.

For all other uses, contact the owner/author(s).

HT '19, September 17-20, 2019, Hof, Germany

(C) 2019 Copyright held by the owner/author(s).

ACM ISBN 978-1-4503-6885-8/19/09.

https://doi.org/10.1145/3342220.3344936

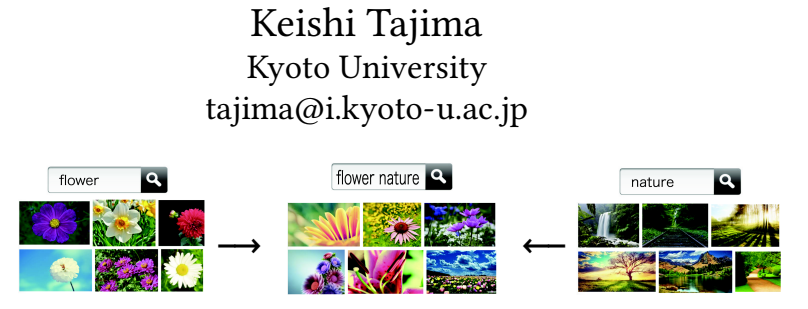

Figure 1: Images of "flower", "nature" and "flower nature".

\section{OUR METHOD}

In this research, we focus on the subject specificity of a tag, i.e., how much the tag narrows down the subjects shown in the associated images. Subject-specificity of a tag is high if all the images associated with the tag shows some specific subjects, and it is low if the tag is associated with images of various subjects. Notice that subjectspecificity is different from the inverse document frequency. Even if a tag appears with many images, if all the images are showing similar subjects, the tag has high subject-specificity.

As explained in Sec. 1, our method compares a pair of tags. Given tags $\mathrm{A}$ and $\mathrm{B}$ of some image, we determine which of them is more subject-specific, and if $A$ is more subject-specific, we determine that $\mathrm{A}$ is more likely to describe the image contents than $\mathrm{B}$ is.

In order to determine which of a given tag pair A and B is more subject-specific, we compare three sets of images: those associated with $\mathrm{A}$, those associated with $\mathrm{B}$, and those associated with both $\mathrm{A}$ and $\mathrm{B}$. If the set with both $\mathrm{A}$ and $\mathrm{B}$ is more similar to the set with $A$ than to the set with $B$, we determine that $A$ is more subjectspecific. For example, Fig. 1 shows the result of image search by "flower" (left), by "nature" (right), and by "flower nature" (center). Most images retrieved by "flower nature" show flowers. It means that the word flower has higher subject-specificity than nature.

In this example, flower is a hyponym of nature, but there are many other types of cases. For example, most images with "apple love" show apples. In this case, the concept of apple is more concrete and can be shown in images more directly than love is. As a result, "apple" has stronger influence on the subjects shown in the images, i.e., has higher subject-specificity. In the case of "flower iPhone", flowers are typical photo subject while iPhone can be a metadata.

In this paper, we compute similarity between image sets not based on their image contents but based on their associated tags. It is because we want to compute conceptual similarity of their subjects, not visual similarity, and text tags better reflect the concepts shown in images. For example, most images with a tag "food" show some food. They include various colors and shapes, but many of them have many common tags, such as dinner and yummy. Experimental comparison with image-based similarity is a future research issue.

To compute similarity between image sets, we first convert each image set into a vector representing word frequency in its associated tags. Each component of the vector corresponds to each word that appears at least once as a tag in the data set. The vector of an image set associated with $\mathrm{A}$, denoted by $V(A)$, is defined as below:

$$
V(A)=\left(\Sigma_{i} c_{A, t_{i}}\right)^{-1} \cdot\left\langle c_{A, t_{1}}, \ldots, c_{A, t_{n}}\right\rangle
$$


where $t_{1}, \ldots, t_{n}$ are the words that appear in the data set, and $c_{A, t}$ is the co-occurrence degree of $\mathrm{A}$ and $t$, which is defined as below:

$$
c_{A, t}=\frac{\left|S_{A t}\right|^{2}}{\left|S_{A}\right| \cdot\left|S_{t}\right|}
$$

where $S_{A}, S_{t}$, and $S_{A t}$ is the set of images associated with A, with $t$, and with both $\mathrm{A}$ and $t$,respectively. Notice that $V(A)$ is normalized by $\left(\Sigma_{i} c_{A, t_{i}}\right)^{-1}$ so that its L1 norm is 1 .

Next we compute similarity between vectors of the three image sets. Cosine similarity is often used for similarity between vectors, but cosine similarity tends to have large values when two vectors have many common non-zero components while we want the similarity to be high when the vectors are similar over all components. We instead use Jensen-Shannon divergence. Let $p, q$ be probability distribution, then Jensen-Shannon divergence between $p$ and $q$, denoted by $J S D(p \| q)$, is defined as follows:

$$
J S D(p \| q)=\frac{1}{2} K L(p \| r)+\frac{1}{2} K L(q \| r)
$$

where $r=(p+q) / 2$ and $K L(p \| q)$ is Kullback-Leibler divergence from $p$ to $q$. Notice that $J S D$ is symmetric while $K L$ is not.

We finally define the similarity between two L1-normalized vectors $V(A)$ and $V(B)$, denoted by $\operatorname{sim}(A ; B)$, as follows:

$$
\operatorname{sim}(A ; B)=1-J S D(V(A) \| V(B))
$$

Table 1 shows the top 10 components of the vectors of the image sets associated with "flower", "nature", and "flower nature". For each image set, we collected 1,000 Instagram posts including the tags. Table 2 shows the similarity between the vector of "flower nature" and the other two. The vector of "flower nature" is more similar to the vector of "flower" than to the vector of "nature", which means the word flower is more subject-specific than the word nature.

\section{EVALUATION}

We ran an experiment to evaluate our method. First, we collected 170 images that have more than three tags from Instagram. Three assessors assigned scores from 0 to 3 to each tag based on whether it is describing the image contents. For each tag, we took the average of the scores by the three assesses, and used it as the ground truth

Next, for each image, we randomly chose a tag with the average score 3 and a tag with the average score 1 or smaller. We determined which tag is more relevant to the image contents by using our method, and compared the result with the ground truth.

We compared our method with a simple baseline method: "A tag appearing earlier in the tag list defined by the user is more relevant". Table 3 shows the accuracy of our method and the baseline. Accuracy of our method is far better than the chance rate 0.5 .

\section{RELATED WORK}

There have been several studies on the estimation of the importance of tags associated to images. Spain and Perona [5] used the order of tags in tag lists associated with images to learn relative importance of objects in images perceived by human users. They focus on relative importance of objects in images, while we focus on whether a tag is describing the contents of the image or some metadata.

Liu et al. [2] and Li et al. [4] ranked tags associated with a image by using a random walk on the graph representing relationship between tags. A disadvantage of their approach is that we have
Table 1: Top 10 Tags of Flower, Nature, and Flower Nature

\begin{tabular}{|l|l|l|l|}
\hline \multicolumn{2}{|l|}{ flower } & \multicolumn{2}{l|}{ nature } \\
\hline bloom & 0.068 & sky & 0.028 \\
floral & 0.062 & landscape & 0.026 \\
botanical & 0.047 & cloud & 0.024 \\
nature & 0.030 & green & 0.022 \\
blossom & 0.029 & outdoor & 0.017 \\
flor & 0.025 & beautiful & 0.016 \\
rose & 0.024 & forest & 0.016 \\
petal & 0.023 & travel & 0.016 \\
spring & 0.022 & mountain & 0.014 \\
summer & 0.021 & grass & 0.014 \\
\hline
\end{tabular}

\begin{tabular}{|l|l|}
\hline \multicolumn{2}{|c|}{ flower nature } \\
\hline bloom & 0.083 \\
blossom & 0.075 \\
petal & 0.061 \\
plant & 0.056 \\
floral & 0.040 \\
green & 0.039 \\
beautiful & 0.037 \\
spring & 0.036 \\
summer & 0.034 \\
forest & 0.032 \\
\hline
\end{tabular}

Table 2: Similarity of Flower/Nature to Flower Nature

\begin{tabular}{|c|c|r|}
\hline$A$ & $B$ & $\operatorname{sim}(A, B)$ \\
\hline flower & flower nature & 0.876 \\
nature & flower nature & 0.550 \\
\hline
\end{tabular}

Table 3: The Accuracy of Our Method and The Baseline

\begin{tabular}{|l|r|}
\hline \multicolumn{1}{|c|}{ Method } & \multicolumn{1}{|c|}{ Accuracy } \\
\hline Our method & $131 / 170(=0.771 \ldots)$ \\
Baseline & $97 / 170(=0.571 \ldots)$ \\
\hline
\end{tabular}

to run a random walk for each image, which becomes a problem when we have a huge image set, such as all images on Instagram.

There have been also several studies that use both textual and visual information for weighting image tags or extracting semantic relationship between tags, such as [1, 3, 6]. An advantage of our method is that we do not need visual information, so our method is highly scalable for huge image sets, and also robust for concepts corresponding to diverse visual characteristics, such as food.

\section{CONCLUSION}

We proposed a method of determining which of a given tag pair $\mathrm{A}$ and $\mathrm{B}$ is more likely to describe not metadata but contents of associated images. Our method determines it by comparing three image sets: those associated with $\mathrm{A}$, with $\mathrm{B}$, and with both $\mathrm{A}$ and $\mathrm{B}$. We ran an experiment on the Instagram data and the result shows that our method is effective. Comparison with the other state-of-art methods is an important remaining work for future research.

\section{ACKNOWLEDGMENTS}

This work was supported by JST CREST JPMJCR16E3, Japan.

\section{REFERENCES}

[1] Marie Katsurai, Takahiro Ogawa, and Miki Haseyama. A cross-modal approach for extracting semantic relationships between concepts using tagged images. IEEE Trans. Multimedia, 16(4):1059-1074, 2014.

[2] Minxian Li, Jinhui Tang, Haojie Li, and Chunxia Zhao. Tag ranking by propagating relevance over tag and image graphs. In ICIMCS '12, 2012, pages 153-156, 2012.

[3] Shangwen Li, Sanjay Purushotham, Chen Chen, Yuzhuo Ren, and C.-C. Jay Kuo. Measuring and predicting tag importance for image retrieval. IEEE Trans. Pattern Anal. Mach. Intell., 39(12):2423-2436, 2017.

[4] Dong Liu, Xian-Sheng Hua, Linjun Yang, Meng Wang, and Hong-Jiang Zhang. Tag ranking. In Proc. of $W W W^{\prime} 09$, pages 351-360, 2009.

[5] Merrielle Spain and Pietro Perona. Measuring and predicting object importance. International fournal of Computer Vision, 91(1):59-76, 2011.

[6] Jinfeng Zhuang and Steven C. H. Hoi. A two-view learning approach for image tag ranking. In Proc. of WSDM 2011, pages 625-634, 2011. 